\title{
Bayesian modeling of volatile organic compound emissions from three softwoods in Hokkaido, Japan
}

\author{
Masaki Suzuki*
}

\begin{abstract}
We used a small chamber method to examine volatile organic compound (VOC) emissions such as a-pinene, $\beta$-pinene, and limonene, from three softwood species in Hokkaido, northern Japan. Tests were conducted for 4 weeks to investigate how the rate of VOC emission changed over time. All VOC emission rates rapidly decreased and could be explained by the sum of two exponential functions. The model was rewritten as a hierarchical Bayesian model to estimate the change in emission rates over time to estimate both intraspecies and interspecies variations. The Markov chain Monte Carlo method was then used to estimate the parameters. Posterior distributions over time were also predicted for VOC emission rates. Then VOC concentrations were simulated using the estimated posterior distributions for a typical room size $\left(30 \mathrm{~m}^{3}\right)$. Our results suggest that high VOC concentrations would shortly occur after installation of wood furnishings, even with adequate ventilation, and that those peak values would exhibit large variations. However, the variations would decrease over time to species-specific VOC concentrations. The model incorporated rather simplified assumptions due to small amount of data used. More intense investigations are needed to gain more accurate and objective predictions.
\end{abstract}

Keywords: Volatile organic compounds, Hierarchical Bayesian modeling, Markov chain Monte Carlo methods

\section{Introduction}

Guidelines and standards for indoor air concentrations of volatile organic compounds (VOCs) have been established in many countries following concerns about indoor air quality. Wood composite materials are identified as a potential source of indoor air pollutants, and their emission behaviors have been thoroughly investigated [1,2]. We had derived a regression model to evaluate acetaldehyde emissions over time from woodbased materials [3]. Solid wood emits terpenes such as $\alpha$-pinene, $\beta$-pinene, and limonene [4]. Some researchers have reported that inhalation of wood oils containing terpenes promotes relaxation [5]. Suzuki et al. [6] revealed that $\alpha$-pinene reduced fatigue in car drivers. However, inhaling large concentrations of terpenes in dwellings may be unhealthy. Exposure to high concentrations of

*Correspondence: suzuki-masaki@hro.or.jp

Forest Products Research Institute, Hokkaido Research Organization, 1-10 Nishikagura, Asahikawa, Hokkaido 071-0198, Japan $\alpha$-pinene [7] and limonene [8] has been shown to cause sensory irritation in mice. Ozone-terpene reactions form potentially toxic air pollutants [9]. An indoor air quality survey of 14 houses in Tokyo (1995-1998) showed that the median concentrations were $15.1 \mu \mathrm{g} / \mathrm{m}^{3}$ for $\alpha$-pinene and $10.0 \mu \mathrm{g} / \mathrm{m}^{3}$ for limonene [10]. A more recent survey (2007-2008), conducted by the same survey team in 13 different houses, found that the median concentrations were $214 \mu \mathrm{g} / \mathrm{m}^{3}$ for $\alpha$-pinene and $53.2 \mu \mathrm{g} / \mathrm{m}^{3}$ for limonene, with a maximum of $3140 \mu \mathrm{g} / \mathrm{m}^{3}$ for limonene. The surveying authors suggested that an increased use of solid woods instead of wood composites or use of alternative solvents instead of regulated solvents is responsible for the higher indoor concentrations of $\alpha$-pinene and limonene in homes [11]. Furthermore, to encourage the consumption of domestic plantation softwoods, the Japanese government has been recently promoting an increased use of wood furnishings in public buildings. Therefore, solid softwood furnishings are expected 
to become more widespread in both public facilities and private living spaces.

Most previous studies have focused on the emission characteristics of adhesive-derived substances, such as formaldehyde from wood composite materials, which are highly industrialized products. In contrast, the emission behavior of solid softwoods used as furnishings has not been thoroughly investigated. Solid wood has a complex tissue structure compared with wood composites. In fact, emission properties of solid wood might widely vary, both within and among tree species. Better insight into VOC concentrations in newly constructed buildings could be obtained with more detailed data on VOC emissions of softwoods over time. Such data would also be useful for isolating the source(s) of terpene concentrations in buildings (i.e., whether they originate from wood or from transient sources, such as consumer products that contain terpenes as a flavoring agent or solvent).

In this study, we examined emissions over time of $\alpha$-pinene, $\beta$-pinene, limonene, and total volatile organic compounds (TVOC) emanating from three common and industrially important softwood species grown in Hokkaido, northern Japan. We employed Bayesian hierarchical modeling to develop robust emission-over-time models that could quantify intraspecies and interspecies variations in emissions. Bayesian modeling describes parameters as probability distributions. This approach can describe arbitrary probability distributions and thus can be efficient when skewed distributions are expected, such as the logarithmic normal distributions that are often observed in measurements of chemical concentrations. Hierarchical modeling has a multi-level structure. In this study, we investigated a two-layer model: one layer describing emission behaviors common to all species, using entire data to avoid species-specific overfitting, the other layer describing species-specific parameters to model variations in individual species using data from individual species. We used the Markov chain Monte Carlo (MCMC) method to obtain model parameters. The Bayesian model and the MCMC method provided both point estimates for model for those parameters. To demonstrate the advantages of using Bayesian predictions to explain variation, we conducted indoor air concentration simulations using the distributions that we derived from the model.

\section{Materials and methods Materials}

We collected five logs of todomatsu (Abies sachalinensis), five logs of karamatsu (Larix kaempferi), and two logs of akaezomatsu (Picea glehnii) from commercial forest plantations; all sample pieces were prepared from heartwood. The logs were sawn to $30 \mathrm{~mm}$ in thickness and kiln-dried.
The specific drying conditions applied to each species to obtain a final moisture content of $15 \%$. Therefore, todomatsu samples were dried at a maximum temperature of $80{ }^{\circ} \mathrm{C}$ for 6 days, the karamatsu samples were dried at a maximum temperature of $85{ }^{\circ} \mathrm{C}$ for 5 days, and the akaezomatsu samples were dried at a maximum temperature of $80{ }^{\circ} \mathrm{C}$ for 6 days. After drying, the sawn samples were planed to a thickness of $22 \mathrm{~mm}$ and then conditioned for at least 2 weeks under a relative humidity of $50 \%$ at $20{ }^{\circ} \mathrm{C}$. We then planed both surfaces of each sample to an additional $1 \mathrm{~mm}$ to obtain fresh emission surfaces and to remove any contamination that might have collected on the wood during the conditioning process. These remaining $20 \mathrm{~mm}$ sections were cut into dimensions of $160 \mathrm{~mm} \times 160 \mathrm{~mm} \times 20 \mathrm{~mm}$. When preparing sections, we carefully avoided large knots ( $>10 \mathrm{~mm}$ in diameter), bark pockets, resin pockets, and reaction wood. After this final preparation of the samples, the samples were tested immediately (within $1 \mathrm{~h}$ ) for their emissions properties.

\section{Small chamber method}

We measured emissions in small (20 L), stainless steel, cylindrical chambers in accordance with protocol established by Japanese Industrial Standard A 1901 [12] and prior research on gaseous emissions from solid wood [13]. We placed the entire measurement system, including the small chambers, into a thermostatically controlled room maintained at $28{ }^{\circ} \mathrm{C}$. Clean air of $50 \%$ relative humidity was supplied to each small chamber using an air supplier (AOE3200, GL Sciences, Tokyo, Japan) at an air exchange rate (air volume ventilated per hour divided by volume of the chamber) of $0.5 \mathrm{~h}^{-1}$. Sample wood pieces were inserted to a sealed stainless steel box with a window of $147 \mathrm{~mm} \times 147 \mathrm{~mm}$ to determine the surface area of emission and to prevent emissions from cross sections and the back side. A pair of boxed sample pieces was placed into each chamber to obtain a loading factor (emission surface area divided by chamber volume) of $2.2 \mathrm{~m}^{2} / \mathrm{m}^{3}$. Two replicate measurements were made for each $\log$ sample. Air sampling was performed on 1, 3, 7, 14 , and 28 days after placing the test pieces into the test chambers. However, some sampling had to be postponed for 1-2 days due to instrumental shortcomings, such as gas chromatography (GC) instrument failure and sampling tube shortage.

\section{Sampling and analysis}

We used a GC to quantify concentrations of $\alpha$-pinene, $\beta$-pinene, limonene, and TVOC emissions. Using an air sampling pump (SP208-100 dual, GL Sciences), the air from chambers was passed through a sampling tube (AERO TD GL-Tube, GL sciences) packed with $0.1 \mathrm{~g}$ of Tenax TA adsorbent resin (20/35 mesh, GL sciences). We sampled 
$1000 \mathrm{~mL}$ of air at a rate of $33 \mathrm{~mL} / \mathrm{min}$ or $300 \mathrm{~mL}$ at $10 \mathrm{~mL} /$ min to avoid the exceeding capacity of the absorbent. We added 100 ng of toluene-d8 (41063-96, Kanto Chemical, Tokyo, Japan) to each sampling tube as an internal standard. These sampling tubes were desorbed in a thermal desorption system at $240{ }^{\circ} \mathrm{C}$ for $10 \mathrm{~min}(\mathrm{CP} 4020$, Varian, CA, USA) attached to a $\mathrm{GC}$ equipped with flame ionization detector (GC-4000, GL sciences). We then separated the desorbed sample in helium carrier gas by a column (RTXVolatiles; $60 \mathrm{~m} \times 0.25 \mathrm{~mm} \times 1.0 \mu \mathrm{m} \mathrm{df}$; Restek, PA, USA) with the following temperature regime: the temperature was increased from 50 to $90{ }^{\circ} \mathrm{C}$ at a rate of $4{ }^{\circ} \mathrm{C} / \mathrm{min}$, from 90 to $250{ }^{\circ} \mathrm{C}$ at a rate of $10^{\circ} \mathrm{C} / \mathrm{min}$, and then maintained at $250{ }^{\circ} \mathrm{C}$ for $14 \mathrm{~min}$. We calibrated $\alpha$-pinene, $\beta$-pinene, and limonene concentrations with the following standards: (+)- $\alpha$-pinene (Aldrich 268070-5G, Sigma-Aldrich), (-)- $\beta$-pinene (Aldrich 402753-1G, Sigma-Aldrich), and $(R)-(+)$-limonene (Fluka 62118-1ML, Sigma-Aldrich), respectively. We calculated TVOC concentrations as toluene-equivalent values based on the total area of all peaks detected between $n$-hexane and $n$-hexadecane using the calibration curve for toluene [14]. Toluene concentration was calibrated using a VOC standard mixture (VOC standard stock solution mix III, 44096-96, Kanto Chemical). The limits of quantification were $3 \mu \mathrm{g} / \mathrm{m}^{3}$ for $\alpha$-pinene, $2 \mu \mathrm{g} /$ $\mathrm{m}^{3}$ for $\beta$-pinene, $5 \mu \mathrm{g} / \mathrm{m}^{3}$ for limonene, and $310 \mu \mathrm{g} / \mathrm{m}^{3}$ for TVOC. All values below the limit of detection (LOD) were replaced with LOD/2 to enable statistical analyses [15].

\section{Emission modeling}

Emission rates were calculated as follows:

$$
R=C \times \frac{n}{L},
$$

where $R$ is the emission rate $\left(\mu \mathrm{g} / \mathrm{m}^{2} / \mathrm{h}\right), C$ is the chamber's air concentration $\left(\mu \mathrm{g} / \mathrm{m}^{3}\right), n$ is the ventilation rate $\left(\mathrm{h}^{-1}\right)$, and $L$ is the loading factor $\left(\mathrm{m}^{2} / \mathrm{m}^{3}\right)$.

To explain how the rate of emission changed over time, theoretical and empirical models (such as mass transfer models [16], power function models [17, 18], and firstorder decay models $[17,18]$ ) were proposed and applied. Chaudhary et al. [19] pointed out that a two-phase model that employs a sum of two exponential functions agreed best with observed emissions in a meta-analysis of 50 published chamber studies of wood and wood composite products. The results of Suzuki et al. $[3,20]$ were consistent with those of Chaudhary et al. [19] when they applied a twophase model for acetaldehyde emissions from wood-based materials. Based on these studies, the following emission model was used:

$$
R=R_{1}+R_{2}=R_{01} e^{-k_{1} t}+R_{02} e^{-k_{2} t},
$$

where $R_{1}$ is the emission rate during Phase $1, R_{2}$ is the emission rate for Phase $2, R_{01}$ and $R_{02}$ are the initial emission rates, and $k_{1}$ and $k_{2}$ are the coefficients that account for the decrease in emission concentrations during Phases 1 and 2, respectively.

Rate $R_{1}$ (with a larger value of $k_{1}$ ) dominates the first emission stage (Phase 1). Over time, $R_{1}$ decreases rapidly and $R_{2}$ (with a smaller value of $k_{2}$ ) dominates (Phase 2). $R_{1}$ is considered to reflect a desorption process from a surface of a material, whereas $R_{2}$ is considered to reflect a diffusion process in a material [21].

We employed Bayesian hierarchical modeling and the MCMC method to determine the parameters of the model. In prior research, parameters were estimated with a least squares method [3]. These methods estimate parameters as points. In contrast, the Bayesian method estimates parameters as probability distributions derived from prior distributions and likelihood using Bayes' theorem, as follows:

$$
p(\theta \mid D)=\frac{p(D \mid \theta) p(\theta)}{p(D)},
$$

where $\theta$ is the parameter of the model, $p(\theta \mid D)$ is the posterior distribution after data $D$ are observed, $p(D \mid \theta)$ is the likelihood of observing $D$ given $\theta, p(\theta)$ is the prior distribution before observation of $D$, and $p(D)$ is a normalizing constant. The normalizing constant is difficult to calculate analytically. The MCMC sampler generates randomized samples from a probability distribution that are proportional to the posterior distribution and thus provides a precise approximation of the posterior distribution [22]. Detailed explanation for the MCMC method is beyond the scope of this paper.

A hierarchical model was constructed for each VOC and TVOC. Each VOC was assumed to have a unique prior distribution of initial emission factors, $R_{01}$ and $R_{02}$. In contrast, $k_{1}$ and $k_{2}$ were assumed to have values that were independent of any particular VOC. This later assumption is supported by Chaudhary and Hellweg's meta-analysis [19], which reports that there are no statistically significant differences in decay rates for various VOCs emitted from the same material nor for the same VOC emitted from different wood products (including solid wood).

If $R_{i j t}$ is the observed emission rate of a certain VOC from test material $j$ of tree species $i$ at time $t(\mathrm{~h})$, then the observed emission rate, including variation among test materials within a tree species, is modeled by the following equation:

$$
R_{i j t} \sim \operatorname{LogNormal}\left(\log \left(R_{01 i} e^{-k_{1} t}+R_{02 i} e^{-k_{2} t}\right), \sigma_{r}\right),
$$


where $\operatorname{LogNormal}(\mu, \sigma)$ is the probability density function of a logarithmic normal distribution with parameters of $\mu$ and $\sigma, R_{01 i}$ and $R_{02 i}$ are the initial emission rates for tree species $i(i=1,2,3)$, and $\sigma_{r}$ is the parameter explaining the variation between individual test materials. To avoid complexity and divergence of the MCMC sampling results, we assumed that parameters $k_{1}, k_{2}$, and $\sigma_{r}$ were not affected by species of tree examined. We modeled the initial emission rates for each tree species, $R_{01 i}$ and $R_{02 i}$, as follows:

$$
\begin{aligned}
& \log \left(R_{01 i}\right) \sim \operatorname{Normal}\left(\log \left(\bar{R}_{01}\right), \sigma_{1}\right), \\
& \log \left(R_{02 i}\right) \sim \operatorname{Normal}\left(\log \left(\bar{R}_{02}\right), \sigma_{2}\right),
\end{aligned}
$$

where $\operatorname{Normal}(\mu, \sigma)$ is the probability density function of a normal distribution with a mean of $\mu$ and standard deviation of $\sigma, \bar{R}_{01}$ and $\bar{R}_{02}$ are the averages for the initial emission rates for all tree species for Phases 1 and 2 (respectively), and $\sigma_{1}$ and $\sigma_{2}$ are the parameters that represent differences among species, respectively. We used a logarithmic transformation to avoid negative values. Weakly informative prior distributions were used for $\bar{R}_{01 i}$ and $\bar{R}_{02 i}$. These prior distributions had lower limits of zero, and upper limits were derived from the literature [13]. The upper limit for prior distributions of emission rates is shown in Table 1.

The prior distributions for $\sigma_{1}$ and $\sigma_{2}$ were non-informative and were automatically set by the MCMC software. Based on background information on acetaldehyde emissions from wood-based products in the literature $[3,20]$ and on statistical results of Chaudhary and Hellweg [19], the value of $k_{1}$ is considered to be somewhere between 0.0 and 0.1 , whereas the value of $k_{2}$ is assumed to be between 0.0 and 0.005 . To achieve this range in values, the specific prior distributions of $k_{1}$ and $k_{2}$ were set as follows [23, 24]:

$$
\begin{aligned}
& k_{p} \sim \operatorname{Normal}\left(m_{\mathrm{kp}}, s_{\mathrm{kp}}\right), \\
& m_{\mathrm{kp}}=\frac{a_{p}+b_{p}}{2}, \\
& s_{\mathrm{kp}}=\frac{b_{p}-a_{p}}{4},
\end{aligned}
$$

where $m_{\mathrm{kp}}$ is the mean of $k_{p}, s_{\mathrm{kp}}$ is the standard deviation of $k_{p}(\mathrm{p}=1,2)$, and $a_{p}$ and $b_{p}$ are the upper and lower

Table 1 Upper limit of prior distributions of initial emission factors

\begin{tabular}{lllll}
\hline & a-Pinene & $\boldsymbol{\beta}$-Pinene & Limonene & TVOC \\
\hline$R_{01}, R_{02}\left(\mu \mathrm{g} / \mathrm{m}^{3}\right)$ & 1000 & 200 & 200 & 2000 \\
\hline
\end{tabular}

limits of $k_{p}$, respectively. With these prior distributions, the probability of that $k_{p}$ is within the assumed intervals is 0.68. We used the statistic program $\mathrm{R}$ (version 3.4.3) [25] and the MCMC sampling software STAN [26] package for $\mathrm{R}$ (Rstan version 2.17.3) [27] to perform MCMC sampling.

\section{Results and discussion Emission rates}

The observed emission rates for of $\alpha$-pinene, $\beta$-pinene, limonene, and TVOC are shown in Fig. 1. The emission rates of todomatsu were lower than the other two tree species. The VOC $\alpha$-pinene was the dominant compound emitted from karamatsu and akaezomatsu woods. Emission rates for most VOCs monotonically decreased throughout the observation period, except for $\beta$-pinene and limonene from some of the tested todomatsu wood samples. Emission rates decreased most rapidly during the 1st week of the study and the rate of emissions steadily slowed over time. After 4 weeks, emission rates of $\alpha$-pinene, $\beta$-pinene, limonene, and TVOC decreased $70-80 \%$ for all species evaluated in the study.

\section{Emission modeling}

Four chains of 3500 samples were generated by the software. The first 1000 steps of each chain were ignored as a warm-up sequence, after which 10,000 MCMC samples were acquired. We confirmed the convergences of all parameters by examining the Gelman-Rubin statistic $\hat{R}<1.1$ [28]. Parameters estimated by the MCMC method are shown in Tables 2 and 3. Estimated emission rates generated from the parameters and curves of the model are shown in Fig. 1. The posterior median was chosen as the representative value because the distributions of parameters were skewed. The light and dark gray areas of Fig. 1 show 95\% and 50\% posterior prediction intervals, respectively. Note that the gray areas show predicted emission rates and not the credible interval of model curves.

The predicted emission rates of akaezomatsu wood varied more widely than the predicted emission rates for the other two tree species. This is due to the wider prediction intervals for $R_{01}$ and $R_{02}$ values, which may reflect the smaller number of samples we tested for akaezomatsu wood. The predicted limonene emission rates for todomatsu varied less than the observed data. This is presumably due to the hierarchical model-specific phenomena called "shrinkage," in which parameters (in this case, $R_{01}$ and $R_{02}$ ) approach the mode of parameters higher in the hierarchy $\left(\bar{R}_{01}\right.$ and $\left.\bar{R}_{02}\right)$ [29]. The degradation model (of emission curves with point estimators of parameters) fit the observed data and 50\% prediction intervals. Equation 2 (with a point estimator of the parameters) 

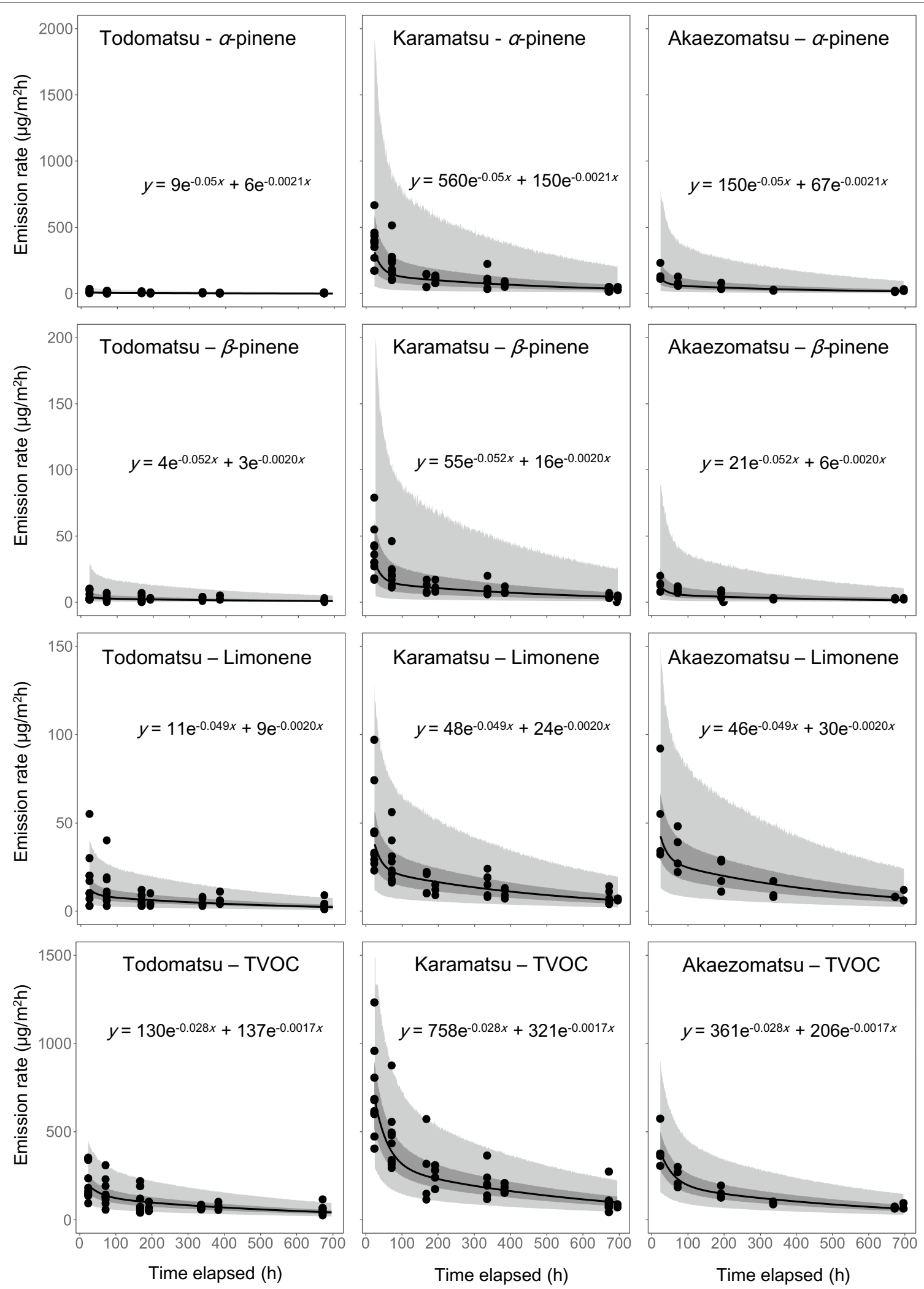

Fig. 1 Relationships between elapsed time and emission factors of VOCs and TVOC. The dots show observed data. Light and dark gray areas show Bayesian prediction intervals of $95 \%$ and $50 \%$, respectively. The black lines show the emission model based on median values predicted at various time intervals 
Table 2 Bayesian $95 \%$ credible interval of $R_{01}$ and $R_{02}$

\begin{tabular}{|c|c|c|c|c|c|c|c|c|c|}
\hline & \multicolumn{9}{|c|}{ Initial emission rates $\left(\mu \mathrm{g} / \mathrm{m}^{2} \mathrm{~h}\right)$} \\
\hline & \multicolumn{3}{|l|}{ Todomatsu } & \multicolumn{3}{|l|}{ Karamatsu } & \multicolumn{3}{|c|}{ Akaezomatsu } \\
\hline & Lower limit & Median & Upper limit & Lower limit & Median & Upper limit & Lower limit & Median & Upper limit \\
\hline \multicolumn{10}{|c|}{ a-Pinene } \\
\hline$R_{01}$ & 1 & 9 & 57 & 6 & 560 & 2291 & 2 & 150 & 1039 \\
\hline$R_{02}$ & 4 & 6 & 9 & 76 & 150 & 240 & 33 & 67 & 116 \\
\hline \multicolumn{10}{|c|}{$\beta$-Pinene } \\
\hline$R_{01}$ & 1 & 4 & 23 & 4 & 55 & 237 & 2 & 21 & 125 \\
\hline$R_{02}$ & 2 & 3 & 5 & 9 & 16 & 25 & 3 & 6 & 11 \\
\hline \multicolumn{10}{|c|}{ Limonene } \\
\hline$R_{01}$ & 1 & 11 & 53 & 5 & 48 & 184 & 2 & 46 & 233 \\
\hline$R_{02}$ & 7 & 9 & 12 & 17 & 24 & 32 & 19 & 30 & 42 \\
\hline \multicolumn{10}{|l|}{ TVOC } \\
\hline$R_{01}$ & 10 & 130 & 408 & 377 & 758 & 2023 & 58 & 361 & 1203 \\
\hline$R_{02}$ & 98 & 137 & 172 & 212 & 321 & 428 & 135 & 206 & 284 \\
\hline
\end{tabular}

Table 3 Bayesian $95 \%$ credible interval of $k_{1}, k_{2}$ and $\sigma_{r}$

\begin{tabular}{|c|c|c|c|c|c|c|c|c|c|}
\hline \multirow{2}{*}{ 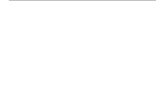 } & \multicolumn{3}{|l|}{$k_{1}$} & \multicolumn{3}{|l|}{$k_{2}$} & \multicolumn{3}{|l|}{$\sigma_{r}$} \\
\hline & Lower limit & Median & Upper limit & Lower limit & Median & Upper limit & Lower limit & Median & Upper limit \\
\hline a-Pinene & 0.014 & 0.050 & 0.101 & 0.0010 & 0.0021 & 0.0029 & 0.76 & 0.87 & 1.00 \\
\hline$\beta$-Pinene & 0.016 & 0.052 & 0.105 & 0.0010 & 0.0020 & 0.0028 & 0.81 & 0.92 & 1.06 \\
\hline Limonene & 0.016 & 0.049 & 0.100 & 0.0013 & 0.0020 & 0.0025 & 0.50 & 0.57 & 0.65 \\
\hline TVOC & 0.012 & 0.028 & 0.078 & 0.0010 & 0.0017 & 0.0022 & 0.35 & 0.40 & 0.46 \\
\hline
\end{tabular}

can be used for obtaining simple point estimates, especially when estimating emissions over a long time scale. Variations can be also estimated with the parameter $\sigma_{\text {r. }}$. Furthermore, the MCMC samples generated with the model contained 10,000 predicted data points for every elapsed hour. This means that emission rates at an arbitrary elapsed hour can be predicted both in a single representative value, such as median, and in the shape of datasets. Using such datasets, the probability of an emission rate being lower or higher than a given value can be easily estimated by summing the number of affirmative values and then dividing the sum by the total number of obtained data points.

With conventional statistics, model parameters are estimated as points. Conventional methods can also estimate prediction intervals, but such methods assume that variations have been generated from normal distributions. Therefore, prediction intervals of emissions may include values less than zero, a nonsensical result relative to emission rates and concentrations. In contrast, the hierarchical Bayesian modeling approach and the MCMC method provide reliable probability distributions for changes in VOCs over time that reflect both interspecific and intraspecific variations. Bayesian modeling explains observed data quite well, enabling one to describe skewed distributions (such as a logarithmic normal distribution) and estimate prediction intervals in VOC emission rates that are positive. In this paper, we used informative prior distributions based on data from previous research. This approach could be subjective, but prior distributions would be supplanted by enough data, and these results tend to converge on the same posterior distribution regardless of different prior distributions [30]. Therefore, more measurements provide more objective and precise estimates of changes in VOC emissions over time.

\section{Simulation of indoor air concentrations}

We simulated changes in indoor TVOC concentrations to demonstrate the advantage of Bayesian predictions and datasets generated by the MCMC method. Because MCMC samples are randomly sampled values generated from posterior distributions of emission parameters, MCMC samples represent VOC emission properties that could arise under the observed conditions and defined prior distributions. Using the MCMC generated sample data in other model functions, new useful 
values (generated quantities) and their distributions can be computed.

When adsorption onto walls and inflow from outdoors is negligible, the mass balance of a test room can be described as follows:

$$
\frac{\mathrm{d} C(t)}{\mathrm{d} t}=L R(t)-n C(t)
$$

where $C(t)$ is the concentration of a VOC at time $t$ (in $\left.\mu \mathrm{g} / \mathrm{m}^{3}\right)$. Substituting Eq. 2 for Eq. 10, assuming an initial VOC concentration of zero, provides the following solution [20]:

$$
C(t)=\frac{L R_{01}\left(e^{-k_{1} t}-e^{-n t}\right)}{\left(n-k_{1}\right)}+\frac{L R_{02}\left(e^{-k_{2} t}-e^{-n t}\right)}{\left(n-k_{2}\right)} .
$$

We already investigated this model by comparing simulation results with actual indoor air concentrationby-time sequences [20]. In this study, acetaldehyde emission rates from wood-based materials, such as plywood and composite floorings, were acquired using the small chamber method. The time series of the acetaldehyde emission rates corresponded reasonably well with the model described by Eq. 2. In addition, simulations of indoor air concentrations were also conducted using Eq. 11 and the parameters of Eq. 2. Then those woodbased materials were put into a real-scale test room. We can reasonably assume that the observed acetaldehyde concentrations in the test room were similar to our simulated results. Because the substances we discussed in this current paper can also be described by Eq. 2, we assume that Eq. 11 could be applied to VOCs as well.

We calculated concentration sequences using Eq. 11 and MCMC samples of $R_{01}, R_{02}, k_{1}$, and $k_{2}$. The simulation condition was a room with $12.7 \mathrm{~m}^{2}$ of floor space,
$2.4 \mathrm{~m}$ of ceiling height, a $0.5 \mathrm{~h}^{-1}$ of ventilation rate (the same conditions as a typically sized room used to test emissions [20]), and $25.4 \mathrm{~m}^{2}$ of todomatsu, karamatsu, or akaezomatsu wood surface. For modeling simplicity, the remainder of surfaces in the room was assumed to emit no VOCs. Predicted TVOC concentrations from time zero to $200 \mathrm{~h}$ are shown in Fig. 2. Because we generated our MCMC samples from posterior distributions of TVOC emission parameters, the predicted concentrations should adequately reflect variations of the TVOC parameters. The predicted concentrations contained no negative values and showed the types of asymmetrical distributions often observed in VOC concentrations. The advantage of using the MCMC method is that it can describe non-normal distributions, unlike estimates of conventional least-square parameters that premise normal distributions. From the predicted concentrationby-time sequences, TVOC concentrations peaked 7-8 h after emissions started. Around the peak period, prediction intervals in TVOC concentrations were wide, reflecting wide variations in $R_{01}$ and $R_{02}$. However, as TVOC concentrations decreased predicted variations contracted (became more narrow). Our simulations suggested that a large variation in initial TVOC emission rates within any given wood species do not affect concentrations over the long term. This means that although excessively high concentrations caused by large variations of initial emission rates can occur in the early stage $(<24 \mathrm{~h})$ of TVOC emissions, TVOC concentrations eventually converge toward a median value, as indicated by the solid black lines in Fig. 2. However, variations among species do affect long-term TVOC concentrations, as depicted with todomatsu and the other two species we examined. The estimated time (at $75 \%$ probability) at which the TVOC concentrations were $<400 \mu \mathrm{g} / \mathrm{m}^{3}$ (the Japanese indoor
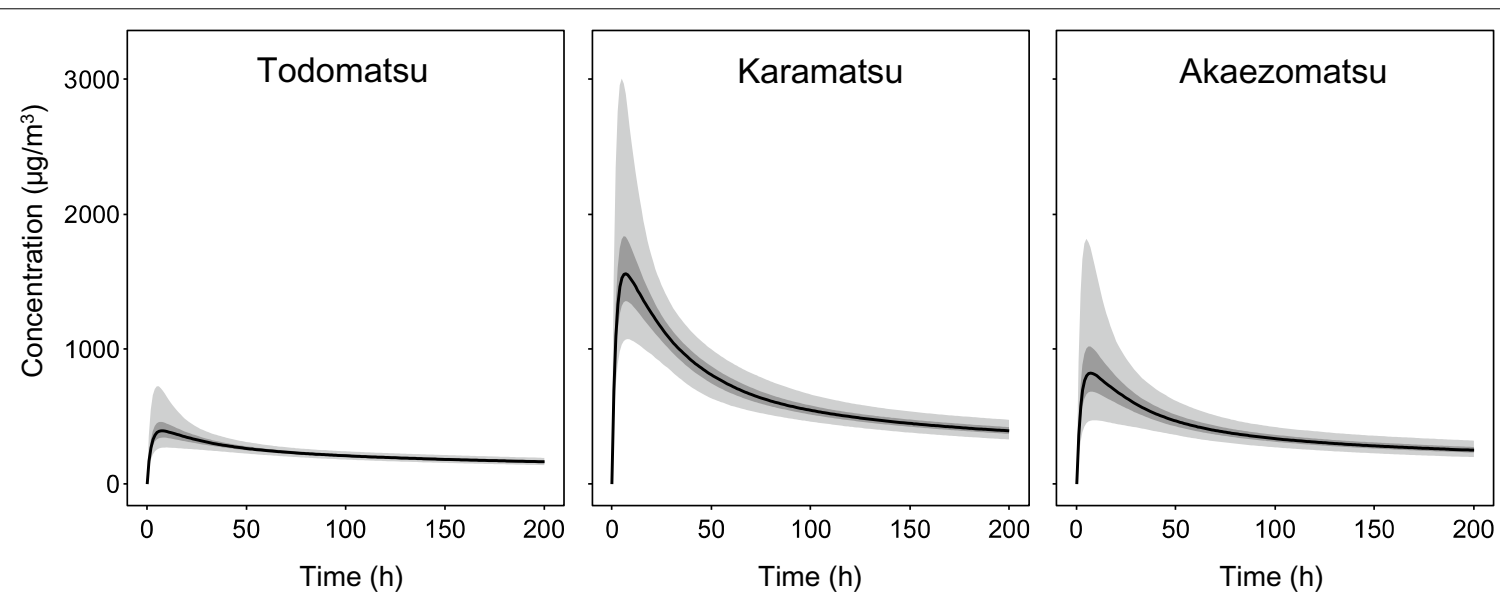

Fig. 2 The simulation results of TVOC concentration sequences, by wood species, for a $30.5 \mathrm{~m}^{3}$ room ventilated at a ventilation rate of $0.5 \mathrm{~h}^{-1}$. Note: Light and dark gray areas depict Bayesian prediction intervals of $95 \%$ and $50 \%$, respectively. The black lines show the median value for predicted concentrations 
air provisional guideline value for TVOC) [31] are $18 \mathrm{~h}$ for todomatsu, $226 \mathrm{~h}$ for karamatsu, and $82 \mathrm{~h}$ for akaezomatsu. Our data on estimated changes in TVOC concentrations over time suggest that even with adequate ventilation, high concentrations of TVOCs occur shortly after a newly manufactured softwood product is introduced into a space; however, TVOC concentrations quickly decline due to both a decrease in the rate of emissions from the newly introduced wood and ventilation of the surrounding space. Therefore, a ventilation rate large enough to dissipate VOCs (e.g., by opening windows) should be recommended for the first $24 \mathrm{~h}$ after softwood products are introduced into a living space. Simulations like the one we used in this study would be useful for estimating the rate at which wood-associated odors are dissipated.

\section{Conclusion}

We investigated $\alpha$-pinene, $\beta$-pinene, limonene, and TVOC emissions from three softwood species harvested in Hokkaido, northern Japan. After 4 weeks in a small chamber, the emission rates of $\alpha$-pinene, $\beta$-pinene, limonene, and TVOC decreased $70-80 \%$ in all three species. We employed a hierarchical Bayesian modeling approach and the MCMC method to estimate changes in the rate of $\mathrm{VOC}$ emissions over time, taking into account both intraspecies and interspecies variations. The decrease in emission rates that we observed was described by a sum of two exponential functions and the parameters acquired the shape of probability distributions. These parameters and prediction intervals were based on both emission behaviors common to all species and species-specific parameters. A room-scaled simulation was performed to predict TVOC concentrations using the obtained models. The models showed that TVOC concentrations would peak at $7-8 \mathrm{~h}$ after placing wood furnishings in a room. At the peak emission period (after initially placing the wood in the chamber), the Bayesian prediction intervals were wide, reflecting high variations in initial emission rates. However, the prediction intervals narrowed over time. In contrast, variations in VOC concentration between species were maintained throughout the simulation period.

\section{Authors' contributions}

MS designed and performed the experiments, analysed the data, and wrote the manuscript. The author read and approved the final manuscript.

\section{Acknowledgements}

The author would like to thank Hokkaido Forestry Cooperative Association for providing specimens, Mr. Akihiro Yakuwa of social welfare corporation Toma Katarubenomori and Mr. Hideaki Tsuchihashi and Dr. Satoru Murakami of Forest Products Research Institute for preparing specimens, Dr. Shinichi Isaji of FPRI for assisting GC analyses, Dr. Shin-ichiro Tohmura of Forest and
Forest Products Research Institute and Dr. Shinichi Kikuchi of Hokkaido Forest Products Technology Promotion Association for their helpful suggestions. This study was funded by FPRI ordinary research (Grant Number 3306-152601).

\section{Competing interests}

The authors declare no competing interests.

\section{Publisher's Note}

Springer Nature remains neutral with regard to jurisdictional claims in published maps and institutional affiliations.

Received: 29 June 2018 Accepted: 8 February 2019

Published online: 22 February 2019

\section{References}

1. Jiang C, Li D, Zhang P, Li J, Wang J, Yu J (2017) Formaldehyde and volatile organic compound (VOC) emissions from particleboard. Identification of odorous compounds and effects of heat treatment. Build Environ 117:118-126

2. Liang $W$, Yang $S$, Yang $X$ (2015) Long-term formaldehyde emissions from medium-density fiberboard in a full-scale experimental room, emission characteristics and the effects of temperature and humidity. Environ Sci Technol 49:10349-10356

3. Suzuki M, Akitsu H, Miyamoto K, Tohmura S, Inoue A (2014) Effects of time, temperature, and humidity on acetaldehyde emission from woodbased materials. J Wood Sci 60:207-214

4. Manninen AM, Pasanen P, Holopainen JK (2002) Comparing the VOC emissions between air-dried and heat-treated Scots pine wood. Atmos Environ 36:1763-1768

5. Miyazaki Y, Motohashi Y, Shigeo K (1992) Changes in mood by inhalation of essential oils in humans I. Effect of essential oils on pupillary light reflex, performance, sensory evaluation and profile of mood states. Mokuzai Gakkaishi 38:903-908 (in Japanese)

6. Suzuki K, Yasuda M, Sassa Y, Harada S (2006) Effects of aroma of trees on active safety during car driving. Trans Jpn Soc Mech Eng C 72:142-150 (in Japanese)

7. Nielsen GD, Larsen ST, Hougaard KS, Hammer M, Wolkoff P, Clausen PA, Wilkins CK, Alarie Y (2005) Mechanisms of acute inhalation effects of (+) and (-)-a-pinene in BALB/C mice. Basic Clin Pharmacol Toxicol 96:420-428

8. Larsen S, Hougaard K, Hammer S, Alarie Y, Wolkoff P, Clausen P, Wilkins C, Nielsen G (2000) Effects of R-(+)-and S-(-)-limonene on the respiratory tract in mice. Hum Exp Toxicol 19:457-466

9. Nazaroff WW, Weschler CJ (2004) Cleaning products and air fresheners: exposure to primary and secondary air pollutants. Atmos Environ 38:2841-2865

10. Saito I, Seto H, Tada T, Nagashima C, Takeuchi M, Tsuchiya Y (1999) The relationship between concentration of indoor air chemicals and the age of houses. Ann Rep Tokyo Metr Res Lab Pub Health 50:235-239 (in Japanese)

11. Onuki A, Saito I, Tada T, Fukuda M, Kurita M, Ogata A, Todoka E, Nakaoka H, Mori C (2009) Trends in indoor air chemicals detected at high concentrations in newly built houses. Ann Rep Tokyo Metr Inst Pub Health 60:245-251 (in Japanese)

12. JIS A 1901:2015 (2015) Determination of the emission of volatile organic compounds and aldehydes for building products: small chamber method. Japanese Standards Association, Tokyo (in Japanese)

13. Ohira T (2004) Aldehydes and VOC emissions from solid wood. In: Forestry and Forest Products Research Institute (ed) Sick building and woodbased materials data book. Tokyo, Ringyo Kagaku Gijutsu Shinkosho, pp 57-73 (in Japanese)

14. JIS A 1965:2015 (2015) Determination of volatile organic compounds in indoor and test chamber air by active sampling on Tenax TA thermal desorption and gas chromatography using MS or MS-FID. Japanese Standards Association, Tokyo (in Japanese)

15. United States Environmental Protection Agency (2000) Guidance for data quality assessment Practical methods for data analysis. Office of environmental information, United States Environmental Protection Agency, Washington DC 
16. Xu Y, Zhang Y (2004) A general model for analyzing single surface VOC emission characteristics from building materials and its application. Atmos Environ 38:113-119

17. Ye W, Won D, Zhang X (2016) Examining the applicability of empirical models using short-term VOC emissions data from building materials to predict long-term emissions. Build Simul 9:701-715

18. Liu X, Mason MA, Guo Z, Krebs KA, Roache NF (2015) Source emission and model evaluation of formaldehyde from composite and solid wood furniture in a full-scale chamber. Atmos Environ 122:561-568

19. Chaudhary A, Hellweg S (2014) Including indoor offgassed emissions in the life cycle inventories of wood products. Environ Sci Technol 48:14607-14614

20. Suzuki M, Hiroshi A, Kohta M, Shin-ichiro T (2016) Estimate of acetaldehyde concentration in a full-scale testing room based on the emission factors of wood-based materials obtained using the small chamber method. Mokuzai Gakkaishi 62:317-324 (in Japanese)

21. Guo H, Murray F, Lee SC (2002) Emissions of total volatile organic compounds from pressed wood products in an environmental chamber. Build Environ 37:1117-1126

22. Kruschke JK (2015) Markov Chain Monte Carlo. Doing Bayesian data analysis: a tutorial with R, JAGS, and Stan, 2nd edn. Academic Press, Cambridge, pp 143-191

23. The Stan Development Team (2017) Prior choice recommendations. https://github.com/stan-dev/stan/wiki/Prior-Choice-Recommendations. Accessed 17 Apr 2018
24. Matsuura K (2016) Weakly informative prior. Bayesian Statistical Modeling Using Stan and R (Wonderful R), vol 2. Kyoritsu Shuppan, Tokyo, pp 184-186 (in Japanese)

25. R Core Team (2018) R: a language and environment for statistical computing. https://www.R-project.org. Accessed 17 Apr 2018

26. Carpenter B, Andrew G, Hoffman MD, Daniel L, Goodrich B, Betancourt M, Brubaker M, Guo J, Li P, Riddell A (2017) Stan: a probabilistic programming language. J Stat Softw. https://doi.org/10.18637/jss.v076.i01

27. Stan Development Team (2018) RStan: the R interface to Stan. http://mcstan.org/. Accessed 17 April 2018

28. Gelman A, Carlin JB, Stern HS, Dunson DB, Vehtari A, Rubin DB (2013) Chapter 11 basics of Markov chain simulation. Bayesian data analysis, 3rd edn. CRC Press, Boca Raton, pp 275-292

29. Kruschke JK (2015) Hierarchical models. Doing Bayesian data analysis: a tutorial with R, JAGS, and stan, 2nd edn. Academic Press, Cambridge, pp 221-264

30. Downey AB (2013) Swamping priors. Think Bayes. O'Reilly Media, Sebastopol, pp 33-34

31. Ministry of Health, Labour and Welfare (2002) Committee on sick house syndrome: indoor air pollution progress report No. 4-Summary on the discussions at the 8th and 9th meetings. http://www.nihs.go.jp/mhlw/ chemical/situnai/kentoukai/rep-eng4.pdf. Accessed 17 Apr 2018

\section{Submit your manuscript to a SpringerOpen ${ }^{\odot}$ journal and benefit from:}

- Convenient online submission

- Rigorous peer review

- Open access: articles freely available online

- High visibility within the field

- Retaining the copyright to your article

Submit your next manuscript at $\boldsymbol{\nabla}$ springeropen.com 